\title{
Introduction to the special issue on "High performance analog circuits and design methodologies"
}

\author{
Luong Mo Dang ${ }^{1} \cdot$ Hideki Asai ${ }^{2}$
}

Published online: 4 September 2015

(c) Springer Science+Business Media New York 2015

This special issue of the SPRINGER Journal of Analog Integrated Circuits and Signal Processing includes a special section composed of four papers related to "High performance analog circuits and design methodologies". They are selected among those presented at the Joint Conference of 4S-2014/2014 International Conference on Analog VLSI Circuits (AVIC 2014).

This conference was jointly organized by Vietnam National University HCMC (VNU-HCM) Integrated Circuit Design Research and Education Center (ICDREC) and Institute of Electrical Engineers of Japan (IEEJ) as an event for commemorating the $20^{\text {th }}$ anniversary of VNU-HCM and 10th anniversary of ICDREC.

The conference is the successor of the former International Analog VLSI Workshop. Its purpose is to exchange information, ideas and recent research results on analog VLSI circuits and their applications.

The first paper, "An Improvement of the CurrentVoltage Conversion Technique in Over-Current Sensing Circuit for Low-Power Low Dropout Linear Voltage Regulators", by Ho Quang Tay, proposes a resistance-less over-current sensing circuit using current-voltage conversion technique for CMOS low dropout linear voltage (LDO) regulators with PMOS as pass device.

The second paper, "Delta-Sigma DAC with Jitter-Shaper-Reducing Jitter Noise", by Yuki Watanabe et al.,

Luong Mo Dang

dangluongmo@gmail.com

Hideki Asai

asai.hideki@shizuoka.ac.jp

1 Integrated Circuit Design Research and Education Center (ICDREC), Ho Chi Minh, Vietnam

2 Shiuoka University, Hamamatsu, Japan describes a novel delta-sigma digital-to-analog converter (DSDAC) using a jitter shaper to augment the noise caused by clock jitter.

The third paper, "Design of a Symmetry-type Floating Impedance Scaling Circuits for a Fully Differential Filter", by Fujihiko Matsumoto et al., proposes the impedance scaling techniques in order to realize a small-size low frequency active filter.

The final paper, "A Low-Power and Low-Gm Linear Transconductor Utilizing Control of a Threshold Voltage", by Takeshi Ohbuchi et al., proposes a linear transconductor that is realized by the scheme that the threshold voltages of the transistors are changed by the attenuated input voltage using body effect and reverse short-channel effect.

On behalf of the editorial committee of this special section, we would like to express our great thanks to all the authors of the submitted manuscripts and all the reviewers for their great contribution and helpful effort. We also would like to thank all of the committee members for their valuable effort expended in this editorial work. Finally, we would like to express our special thanks to the Guest Editorial Secretary, Professor Cosy Muto, Nagasaki University and Professor Hiroo Sekiya, Chiba University, and the Advisor, Professor Takeshi Shima, Kanagawa University, for all their contributions and efforts. 


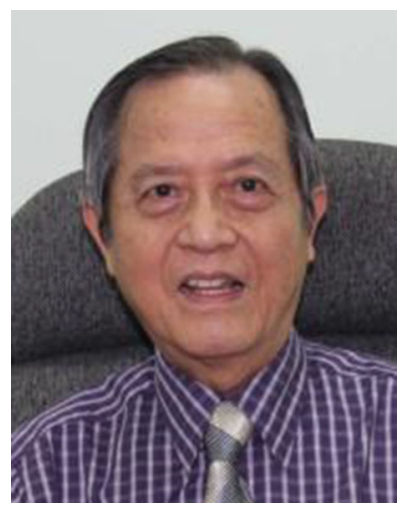

Luong Mo Dang was born in Viet Nam but received higher education in Japan. He received the degrees of B.E., M.E., and Dr. Eng. from The University of Tokyo, Tokyo, Japan, in 1962, 1964 and 1968, respectively. After a year of Post-Doctoral research, also at The University of Tokyo, he joined Toshiba Central Research Laboratories (presently Toshiba R\&D Center) in 1969 where he was engaged in the research and development of semiconductor devices. From 1971 to 1976, he was associated with the Saigon University of Science (presently Ho Chi Minh University of Science) of Viet Nam, and then with the National Institute of Technology of Viet Nam (presently Ho Chi Minh University of Technology), where he served as Rector in 1973-1975. He returned to Toshiba in July, 1976, then moved to HOSEI University, Tokyo, Japan, in April 1983, as Professor of Engineering and Head of the newly established Department of Electronics and Information Technology. He retired from HOSEI University and came back to Viet Nam in 2002. Some of his academic achievements include the Dang Model for short-channel effect, which is embedded in the MOS Model Level 3 of the world renowned circuit simulator SPICE, the two-dimensional and threedimensional numerical research of various effects of the short-channel and narrow-channel MOS transistors. In 2005, he established the Integrated Circuit Design Research and Education Center (ICDREC) in the Viet Nam National University of Ho Chi Minh City (VNUHCM) and serves as its Senior Advisor ever since. The Center succeeded in the design and trial fabrication, in 2007, of the first 8-bit microprocessor (SIGMAK3) of Viet Nam, which was hailed as an event that marked the entry of Viet Nam in the world chip market. He is decorated with the Order of Labor Glory by the President of Viet Nam in recognition for his contribution to higher education and the development of the semiconductor IC chip industry of Viet Nam.

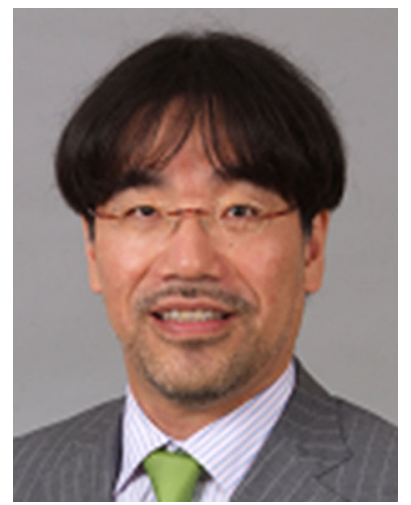

Hideki Asai (IEICE Fellow) received the B.E., M.E., and Ph.D. degrees in electrical engineering from Keio University, Yokohama, Japan, in 1980, 1982, and 1985, respectively. He was with the Department of Electrical and Electronics Engineering, Sophia University, Tokyo, Japan, in 1985. He was a Visiting Professor with Carleton University, Ottawa, ON, Canada, and Santa Clara University, Santa Clara, CA, from 1999 to 2000. Since 1986, he has been with Shizuoka University, Hamamatsu, Japan, where he is currently a Professor involved with VLSI-CAD/CAE and electrical design automation, analog circuit design, and neural networks. He has authored Exercise Notes of Digital Circuits (Corona Publishing Co., Ltd., 2001) and Electronic Circuit Simulation Techniques (Kagakugijyutsu Publisher, 2003). Dr. Asai is a member of the IEEE Nonlinear Circuits and Systems Technical Committee. He was a Chairman of the Technical Group on Nonlinear Problems of IEICE from 2007 to 2008, and the Technical Group on System Packaging CAE of JIEP from 2007 to 2009, and was an Executive Board Member of JIEP. He was a general chair of the EDAPS2013. He received the Prize for Science and Technology (Research Category) awarded by the Minister of Education, Culture, Sports, Science and Technology, the Takayanagi Anniversary Award, in 2009 and the IEICE Fellow, in 2013. 